\title{
Electric Sasando of East Nusa Tenggara, Indonesia
}

\section{Yohanes Don Bosko Bakok}

Catholic University of Widya Mandira, Jenderal Achmad Yanni Street No. 50-52, Kupang, Indonesia ,Email: jokobakok@yahoo.com

\begin{abstract}
Sasando is a stringed musical instrument from Rote Island, East Nusa Tenggara, Indonesia. Based on the resulting tones, this instrument consists of two types namely gong sasando that produces pentatonic tones and violin sasando that produces diatonic tones. Based on these two kinds of sasando the electric sasando has been created with the consequence that some of original characteristic of accoustic sasando had been eliminated. Up to this point, a question is raised, why the electric sasando was created and how is its history? To analyze this problem I use the popular culture studies to indicate that the creation of electric sasando is closely linked with consumer demand, that influenced by popular culture. The datas are obtained through direct observation and by interviewing the informans. Result sows that the creation of electric sasando is influenced by consumer demand who live in popular and mass culture.
\end{abstract}

Keywords: Electric sasando, accoustic sasando, uniqeness, change, popular culture.

\section{INTRODUCTION}

Indonesia is a country that has many different types of art. Some among them still survive in its authenticity, but some are already changed. Factors contributing to the change include the entry of foreign cultures into the local cultures and assimilate with them. The art is finally growing in a new form, as a result of the combination of the original and new elements. Another factor is the development of popular culture that changes the orientation of modern people from the substantial aspects of art to the artificial aspects and prefer to develop the image and style. In this paper I will describe about the art from East Nusa Tenggara (NTT), Indonesia which is the art of music, especially musical instrument named sasando. This stringed instrument initially have a fairly simple form, the number of strings also very limited, but from time to time continue to grow and change. One manifestation of these changes is the emergence and development of electric sasando which on the one hand has enriched the musical treasures of sasando, but on the other hand eliminates certain elements which characterizes the sasando instrument. Based on this phenomenon then appeared a few problems here. Why the electric sasando was created and developed untill now? How is the history of electric sasando?

The aim of this research is to find out the reason of creating electric sasando and to investigate its history. The development of electric sasando can not be separated from the influence of popular culture today that demands sasando to compete in it. 
Pop culture is closely related to the mass culture that is influenced by the spirit of consumerism and the appeal of commodities. This is the trend in today's social reality that can not be stemmed unless faced with a dialogical approach (Strinati, 2009). The study of popular culture would lead us to an understanding of the reasons why traditional arts currently changing as it occurs in sasando musical instrument in NTT.

\section{MATERIALS AND METHODS}

The method used in this research is qualitative method. Datas are obtained through direct observation and by interviewing the informans. The informans consist of the electric sasando maker, sasando teacher and player, NTT leading figure who knows well about the history of electric sasando. The questions asked to the informans were focused on the reason of creating the electric sasando and its history. Some additional question asked is about the original sasando to be compared with the electric one. The collected datas are completed with the informations quoted rightly and with permition from resources books and internet website.

\section{DISCUSSION}

A General Overview of Sasando.

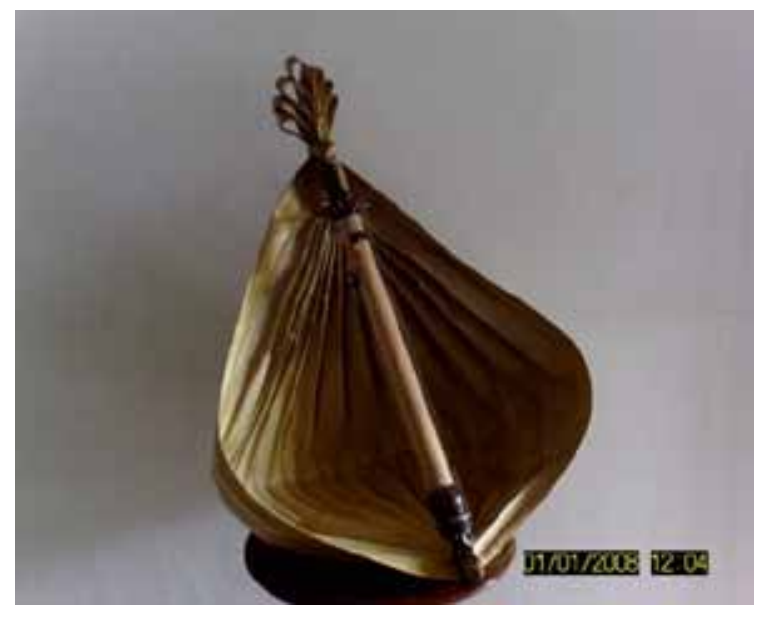

Figure 1. Gong Sasando. (Photo: Edon Sasando)

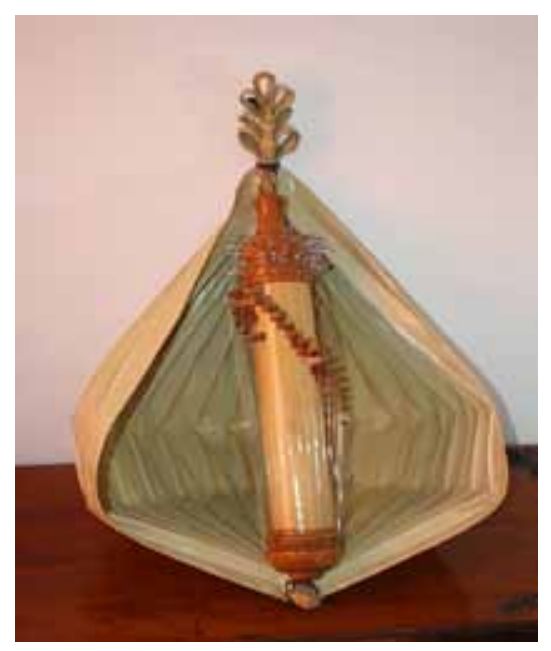

Figure 2. Violin Sasando (Photo: Edon Sasando) 
Sasando comes from the island of Rote, East Nusa Tenggara, Indonesia. Most residents of this island are farmers while those who live on the coast working as fisherman. In ancient times, when the island's population still account for a bit, most of the land overgrown with palm trees named lontar. Leave of lontar is then used as a resonator for acoustic sasando. The emergence of sasando for the first time and the one who found it until now could not be known clearly. Among the people of Rote themselves developing multiple versions of the story about the inventor of sasando. The first version relates that sasando first made by Sangguana, a Rote young man who then gave it to the princess in the Nusaklain palace. Another version tells that sasando for the first time made by the two friends, Lunggi Lain and Balok Ama Sina who worked daily as shepherd and wine tapper (Theedens, 1996). The third version relates that sasando for the first time discovered by Pupuk Soroba inspired by a spider's web (Haning, 2009).

Among Rote people, sasando is a special instrument. Not everyone can play this instrumen because there is a certain poetic verses that should be sung by the players when playing it. The poetic verses containing important teachings to Rote people. When sasando started playing, people around the player should listen in the silence so that the messages of the teaching could be listened and kept in their mind and heart and the sound sasando could be listened clearly. Bamboo that is used to make sasando is the special bamboo, as well as lontar leave that is used as the resonator. Bamboo and the leave must be the best and are taken in a particular moment anyway.

First made sasando is gong sasando because the tones it produces imitate the sound of Rote gong. Number of strings is still limited, only between 9-11 strings and producing pentatonic tones. Strings are tied on small nails that is attached to a bamboo tube on the top and bottom sides. Between the strings and the bamboo tubes there is bridge made of wood pieces that are arranged in such a way according to the high and low of the tones. Beside gong sasando, further developed types of sasando is violin sasando. It is called violin sasando because the tones it produces are diatonic tones imitates the tones found on the violin, although its number is limited. The number of strings had increased to 32 and 36 strings.

The last type of sasando developed from gong sasando and violin sasando is electric sasando. Actually, electric sasando is not a new species of sasando. It is just an electric kind of gong and violin sasando that is made to get a greater volume and clear-varied sound. In addition, there is an acoustic sasando which also uses electrical devices with the same purpose. In the bamboo tube of acoustic sasando a transducer is placed so that the volume is enlarged and the original sound of the instrument is still maintained. In other words, the original sound of sasando in acoustic sasando is unchanged while in the electric sasando the sound is converted to electrical sound. The variety of sounds can be produced by using the sound effect tool. So, it can produce the sounds of the organ, violin, flute and other instruments as we can find in electric guitar using the sound effect tool. 


\section{Electric Sasando}

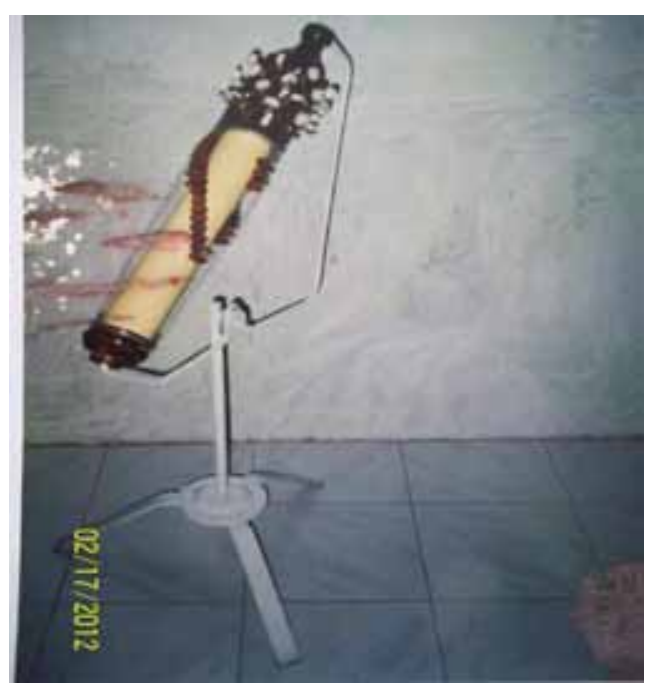

Figure 3. Electric sasando in 1980 (Photo: Edon Sasando)

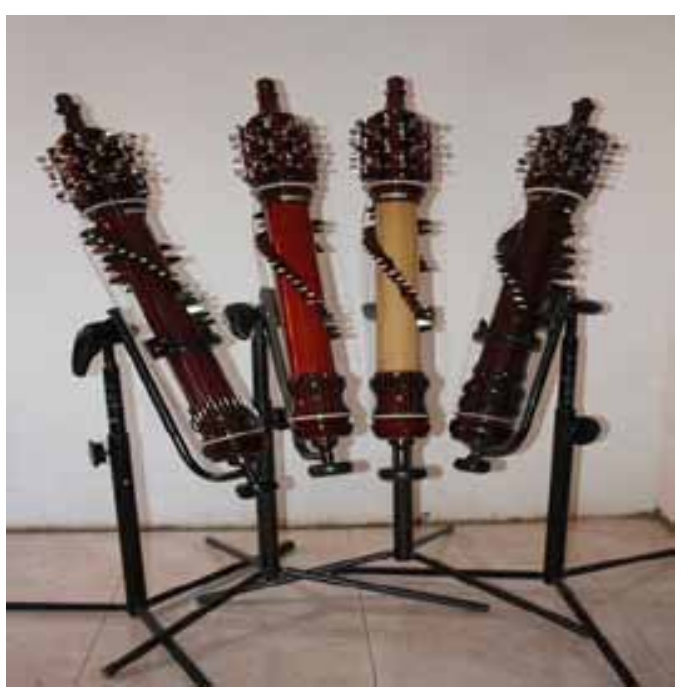

Figure 4. Electric sasando in 2012 (Photo: Edon Sasando)

\section{The Inventor of the Electric Sasando}

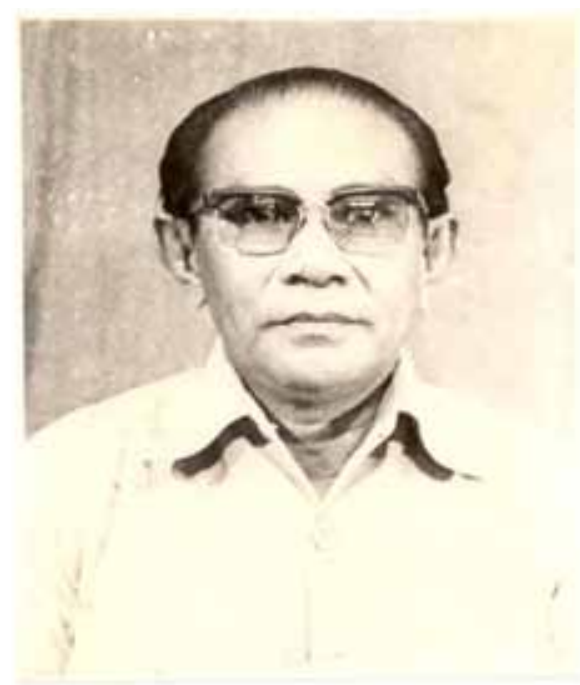

Figure 5. Arnoldus Edon (1923-1994), inventor of electric sasando.

(Photo: Edon Sasando)

The initial idea of making electric sasando driven by the need for a sasando with larger volume and clearer sound. Previously, the effort to increase the volume of sasando is performed by using a microphone that is placed near the sasando but the sound of the strings is not banalnced between melody, rhithm and bass sections and the friction sound of the instrument and other sounds around it also sounded. Under these conditions it was late Arnoldus Edon in 1958 began conducting experiments to make electric sasando. He was a physics teacher, was born in Oesao, Kupang dated August $28^{\text {th }}, 1923$. As the result of the experiment in 1960 he successfully created the first electric Sasando. In the following years he continued to conduct experiments to create a better electric sasando from the aspect of sound quality and design of the instrument. 
During his life he has created 350 electric sasando based on consumer demand as well as on his own initiative. On December $20^{\text {th }}, 2008$, at the $50^{\text {th }}$ birthday of the NTT province, late Arnoldus Edon received an award plaque from the governor of NTT as the inventor of electric sasando.

When Arnoldus Edon died in 1994 his work was continued by his son, Caro David Habel Edon. David himself had learned to make electric sasando from his father since 1972 when he was 10 years old. This time he also continued to make experiments using the tools of new technology in the field of electronics. Of his own hand, he already produced 100 electric sasando. In addition to electric sasando, he also make gong and violin acoustic sasando, using pick up and resonator. He even can make electric and acoustic sasando at once in the same instruments. He make the electric sasando by putting pick up inside the bamboo tube and conect it by a jack cable to the speaker. He himself rafts the pick up using electric wire coil according to the size of bamboo tube. Besides David, there are also some other electric sasando makers in NTT such as Djony Theedens, Yeremias Pah and Oak Zakarias Ndaong. Each sasando they produce has different design and tuning system.

\section{The Organology of Electric Sasando.}

In terms of organology, electric sasando is a stringed musical instrument that consists of several parts. The Folowing are the parts of Edon electric sasando.

a. Upper part.

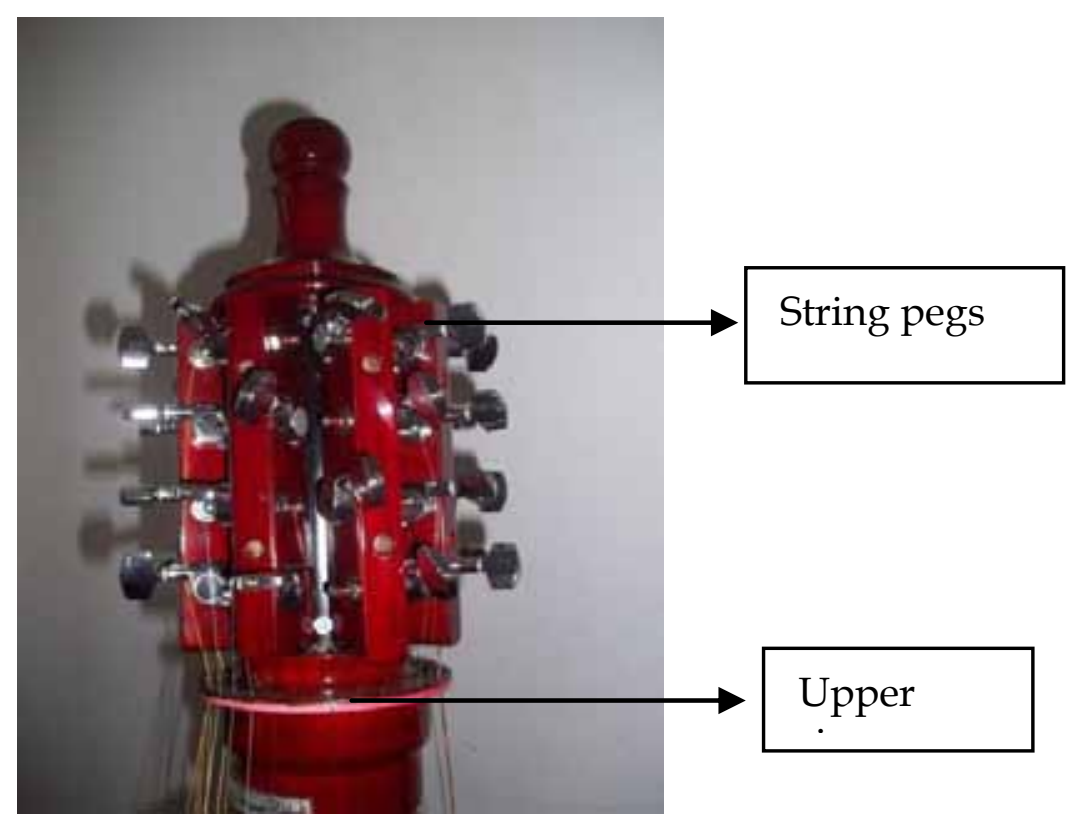

Figure 6. Upper part of electric sasando (Photo: Edon Sasando)

The upper part, called the head of sasando, is the part where string pegs are placed. At the bottom of sasando head there is a ring with small holes to put the string. 
b. Middle part.

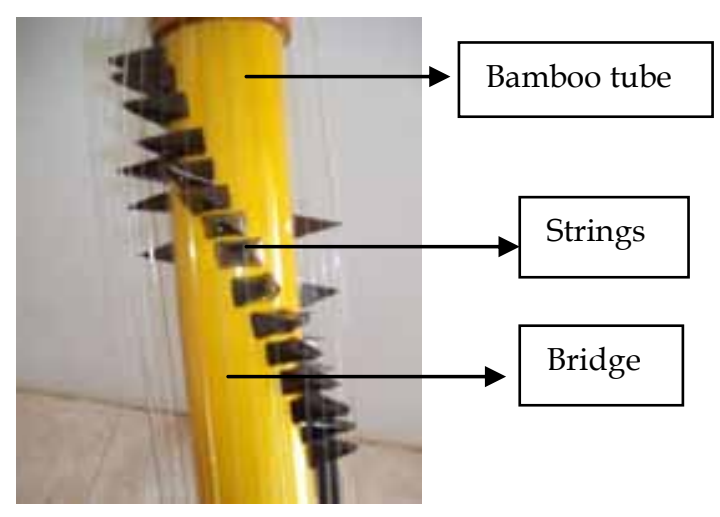

Figure 7. Middle part of electric sasando. (Photo: Edon Sasando)

The middle part of electric sasando consists of a bamboo tube, length $30-50 \mathrm{~cm}$, diameter $5-8 \mathrm{~cm}$. In between the string and bamboo tube there are bridges that hold the strings. The amount is equal to the number of strings.

c. Lower part.

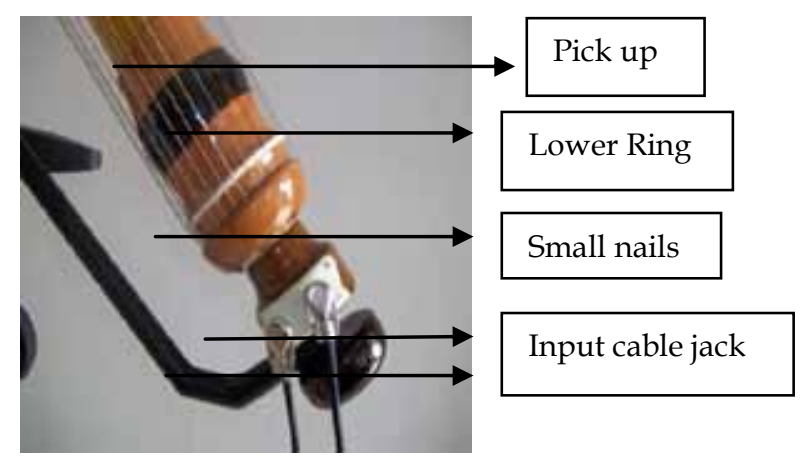

Figure 8. Lower part of electric sasando. (Photo: Edon Sasando)

At the lower part of electric sasando there is also a ring to place the strings and the small nails where sasando strings are tied.

d. The Last Part.

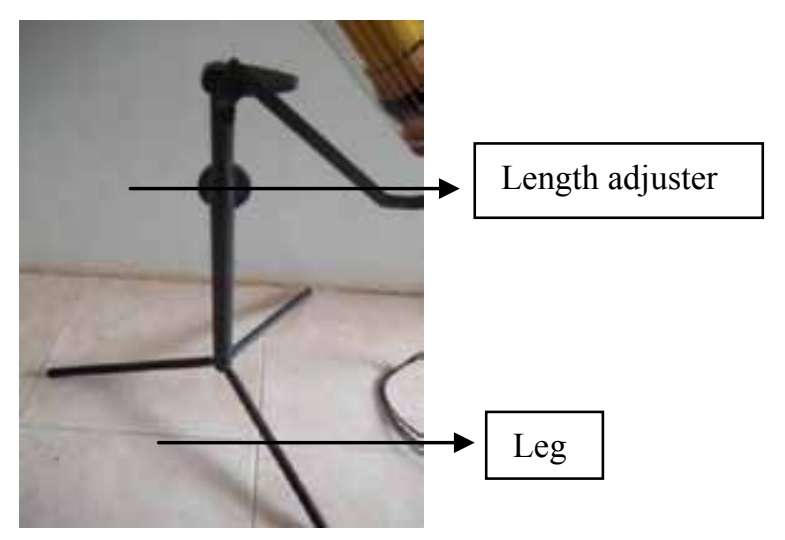

Figure 9. The last part of electric sasando. (Photo: Edon Sasando) 
Electric sasando is equipped with pole made of metal. Initially, the pole is made of two bended big wire in order to sustain sasando on the table, but later is developed to a pole with three legs. The latter kind of pole is still used to this day, although there is a little change in shape and size.

\section{Playing Technique of Electric Sasando.}

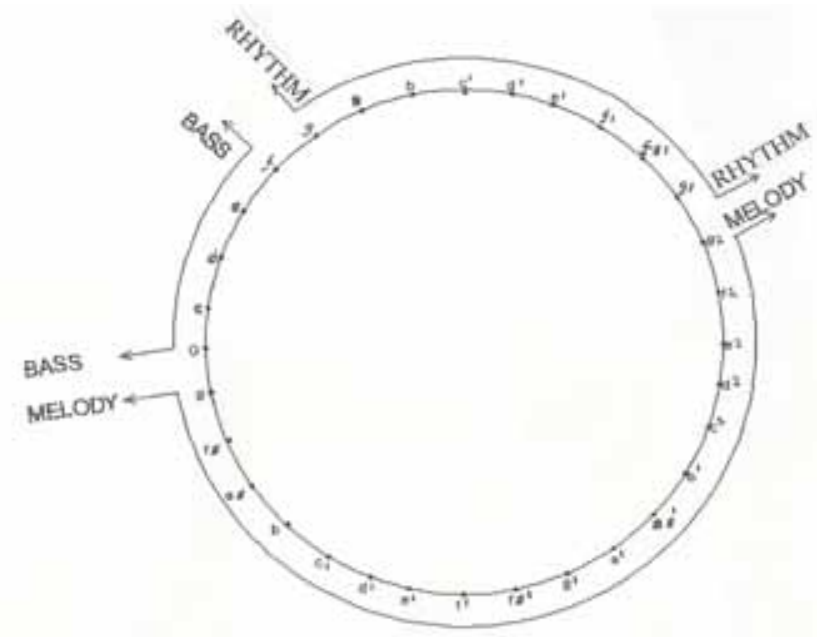

Figure 10. The String Arranggement of Electric Sasando (Sketch: Edon Sasando)

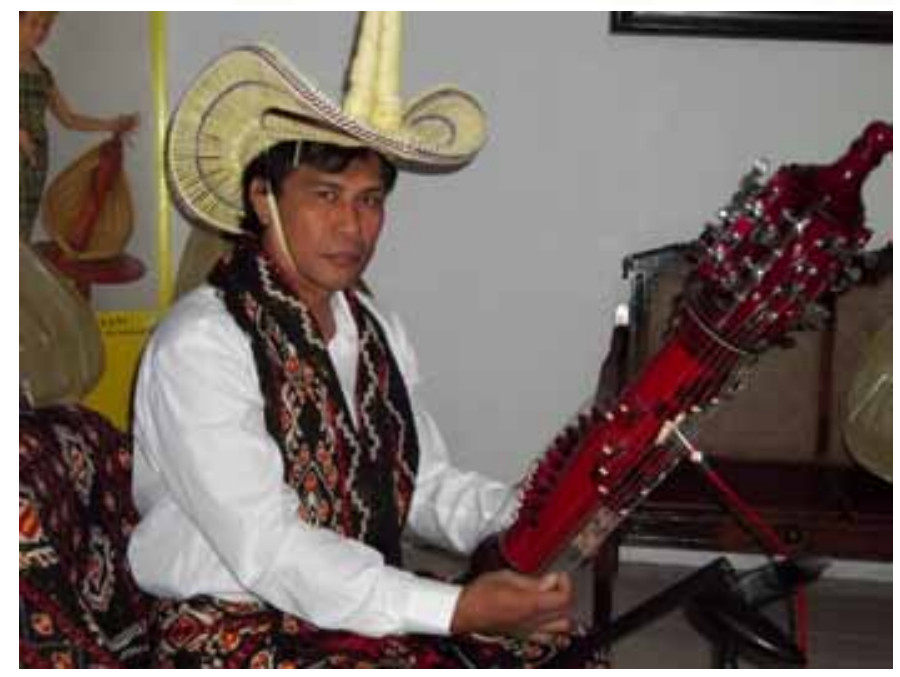

Figure 11. Caro David Habel Edon is playing his electric sasando (Photo: Edon Sasando)

This paper only shows the playing technique of Edon electric sasando. Up to now, on Edon electric sasando we could only play two scales namely $\mathrm{C}$ major and $\mathrm{G}$ major. David is currently finishing his electric sasando with 40 strings that can be played in 4 scales.Electric sasando, as gong and violin sasando, are generally played in seating position but can also be in standing position by setting the length of pole in the proper position.

Electric sasando can be played in solo or a group form. Left hand fingers that are used to play sasando are thumb, index finger, middle finger and ring finger. Thumb functions to play the melody, the index as a stabilizer finger, the middle and ring fingers is used to play the bass strings. Right hand fingers that are used to play are the thumb, index and middle fingers. All this three fingers are used to play rhythm 
according to particular rhythm pattern. In the scale of $C$ major, middle and index fingers of the right hand also play bass in $\mathrm{d}$, e and $\mathrm{f}$ strings for ii, iii and IV chords. The strings that are played to the melody ranging from g to g2 (see sketch above). Bass ranging from $G$ to $f$ strings, rhythms ranging from $g$ to $d 1$ strings.

a. The playing technique of electric sasando in the scale of C Major.

When playing chord I (C major), the ring finger of left hand plucks c string, while the middle finger of the right hand plucks $g$ string, index finger $\mathrm{c} 1$ string and thumb e1 string. When playing chord ii (D minor), ring finger of the left hand plucks $d$ string for bass, middle finger of the right hand plucks $\mathrm{f}$ string, index finger a string, thumb $\mathrm{d} 1$ string. When playing chords iii (E minor), ring finger of the left hand plucks $G$ string for bass (second inversion), while middle finger of the right hand plucks the e string, index finger $\mathrm{g}$, thumb $\mathrm{b}$. When playing chord IV (F major), ring finger of the left hand plucks c string for the bass (second inversion), while the middle finger of the right hand plucks $f$ string, index finger $a$, thumb c1. When playing the chord V (G major), the ring or middle finger of left hand plucks $G$ string, middle finger of the right hand plucks $g$ string, index finger $b$, thumb $d 1$. When playing chord vi (A minor), ring finger of the left hand plucks c string for the bass (first inversion), while the middle finger of the right hand plucks the a string, index finger $\mathrm{c} 1$, thumb e1. When playing chord VII (B diminished), the ring finger of left hand plucks $d$ string for bass (first inversion), the middle finger of the right hand plucks the string $f$, index fingger $b$, thumb d1. At all of these chords, melody is played by the thumb of left hand.

b. The playing technique of electric sasando in the scale of G Major.

When I playing chord I ( $G$ major), middle finger of the left hand plucks $G$ string for the bass, while middle finger of the right hand pluck $g$ string, index finger $b$, thumb d1. When playing chord ii (A minor), ring finger of the left hand plucks c string for the bass (first inversion), middle finger of the right hand plucks a string, index finger $\mathrm{c} 1$, thumb e1. When playing chords iii (B minor), ring finger of the left hand plucks $\mathrm{d}$ string for bass (first inversion), middle finger of the right hand plucks $b$ string, index finger $\mathrm{d} 1$, thumb $\mathrm{f} \# 1$. When playing chord IV (C major), ring finger of left hand plucks c string for bass, middle finger of the right hand plucks g string, index finger $\mathrm{c} 1$, thumb e1. When playing chord V (D major), ring finger of the left hand plucks $d$ string for bass, middle finger of the right hand plucks a string, index finger $\mathrm{d} 1$, thumb $\mathrm{f} \# 1$. When playing chord vi (E minor), ring finger of the left hand plucks $G$ string, middle finger of the right hand plucks e string, index finger $\mathrm{g}$, thumb b. When playing chord VII (F\# diminished), ring finger of the left hand plucks c string for bass (second inversion), middle finger of the right hand plucks string a, index finger $\mathrm{c} 1$, thumb $\mathrm{f} \# 1$.

c. The steps in learning electric sasando.

c.1. Melody learning. Left thumb playing scale of $C$ Major on the melody section separately ranging from $g$ to $g 2$. Focus firstly on the notes of the $C$ Major scale (c, d, e, f, g, a, b, c) then began to memorize the position of each string. Note that between the $\mathrm{f} 1$ and $\mathrm{g} 1$ string there is $\mathrm{f} \# 1$ string and between $\mathrm{a} 1$ and $\mathrm{b} 1$ string there 
is a\# string, as well as at the lower octave, between the $\mathrm{g}$ and $\mathrm{b}$ string there are $\mathrm{f \#}$ and a\# strings. These strings are used only for certain melody, especially for the melody in $G$ scale. Next, try playing a simple melody without rhythm and bass accompaniment so that the left thumb used to pluck the melody, begin with a slow tempo and then accelerated.

c.2. Rhythm learning. Middle finger, index finger and the thumb of the right hand separately play some rhythm patterns and arpeggios (the fingers pluck the strings in sequence, not simultaneously) according to the chords above.

c.3. Melody and rhythm learning simultaneously. Left thumb playing simple songs accompanied by the rhythm of the left hand. First play the songs accompanied by the rhythm of three major chords (I, IV and V) then use the other chords.

c.4. Bass learning. Ring finger and middle finger of the left hand alternately plucking strings $\mathrm{c}$ and $\mathrm{G}$ without melody and rhythm, begin in a slow tempo further getting faster.

c.5. Exercise bass and rhythm simultaneously.

c.6. Exercise bass, rhythm and melody simultaneously. At this step, the player must be able to focuse on these three sections to produce a harmonious blend.

\section{Advantages and Disadvantages of Electric Sasando}

Those who concern in sasando give an assessment that, when compared with the acoustic sasando, electric sasando has some advantages and disadvantages. One of its advantages is it is able to produce high volume and clear sound. If using sound effects then it can produce many more varied sound. Thus, electric sasando is played in the way and position of sasando but has advantages in terms of volume and clarity of sound. Another advantage of electric sasando is that electric sasando is no longer use palm leaves as a resonator that hinders the audience to see the beauty of fingers movement of the players.

Moreover resonator made of palm leaves are very susceptible to weather and collisions that could cause damage. Its disadvantages are, although the resulting volume is much higher and clearer, but the sound is no longer resembles the original sound of sasando. From the aspect of physical appearance and the way how to play, the instrument is realy a sasando but the sound character exactly the same with an electric guitar, whereas the main character of sasando music lies in the sound, as the main material of music is sound. Another disadvantage of electric sasando is not using the resonator made of palm leaves. By using the resonator of palm leaves sasando actually look more natural and artistic. So, there are at least two aspects left of original instrument, the sound character of sasando and the palm leaves as the resonator.

\section{CONCLUSION}

The electric sasando currently had passed through a long historical process. Its being in the history is inseparable from the existence of acoustic sasando which have emerged and developed in the previous periods. The idea of making this instrument is based on the need for a sasando instrument that has a high volume and clear sound and can be combined with the other pop music instruments. Base on physics knowledge, Arnoldus Edon tried to conduct experiment by experiment to fulfill those needs. Finally in 1960 he successfully created the first electric Sasando. Over 
time the development of these instruments continue to evolve in terms of design and sound quality, based on experiments that is accord to the technological advances in the field of electronics. Thus, electric sasando is an innovation, as a result of the development of the sasando instrument preexisting. The new discovery has gained public acknowledgment with the capitulation of the award to late Arnoldus Edon as the inventor of the electric sasando by the governor of NTT. He was deserve to get the award because he had been preserve the sasando musical instruments and the public has been enjoying his work.

There are two different reactions of performers and audiences concerning the development of the electric sasando, some agree but the others not. Those who agree suggested that the electric version is more comfortable with powerful sound and suitable to be played with the other popular musical instruments. Those who disagree suggested that electric sasando is no more deserve to be called sasando because the sound character is already changed and physically lost its uniqueness. Thus, the efforts to compare electric sasando with acoustic sasando is an irrelevant deeds. Electric sasando need not to be compared with the acoustic sasando to identify which one is better because they are different and unique. Differences that exist between the two is a factor that enriches the sasando musical treasures.

Now electric sasando being developed and widespread in both national and international scope to meet consumer demand driven by pop culture. The order of the consumer for this instrument is increasing day by day and currently more young people learning it. It can not be denied that both producers and consumers have been involved in the world of commodities in which the aesthetic aspect of the sasando music itself no longer be considered. It was apparent from the fact that the original characteristic of sasando sound and other accessories such as resonator of palm leaves has been eliminated and more developed the electric sound color to fulfill the consumer demand in market relations. This is the reality in the popular culture that cannot be rejected unless accepted by the dialogical and critical notion.

\section{REFERENCES}

Haning, P. (2009). Sasandu, Alat Musik Tradisional Masyarakat Rote Ndao, Kupang, Kairos.

Strinati, Dominic. (2009). Popular Culture, Yogyakarta: AR-RUZZ MEDIA.

Theedens, D. (1996). Pedoman Bermain Sasando, Kupang, CV Pengharapan Karya Abadi.

\section{ONLINE SOURCE:}

http://edonsasando.wordpress.com/2011/12/02/perkembangan-sasando-elektrik_02/

\section{INTERVIEWS:}

Ady Dethan, 39, Oebufu, Kupang, NTT.

Ben Mboi, 78, Cilandak, Jakarta South.

Caro David Habel Edon, 51, BTN Kolhua, Kupang, NTT.

Djony Theedens, 56, Naikoten 1, Kupang, NTT.

Marline F. Meyners, 47, BTN Kolhua, Kupang, NTT.

Melkior Kian, 46, Oesapa, Kupang, NTT. 